\title{
Un recorrido por las experiencias de trabajo asociativo autogestionado en el sur de México*
}

\author{
María Amalia Gracia** \\ Jorge Enrique Horbath Corredor*** \\ Recibido: 2013-08-20 Aprobado: 2013-I0-I2 Disponible en línea: 2014-05-30 \\ doi:10.III44/Javeriana.CDR.II-73.reta \\ Cómo citar este artículo: Gracia, M. A. y Horbath-Corredor, J. E. (20I4). Un recorrido por las experiencias de \\ trabajo asociativo autogestionado en el sur de México. Cuadernos de desarrollo rural, II(73), 171-190. doi:10.1II44/ \\ Javeriana.CDR $\mathrm{II}-73$.reta
}

\section{Resumen}

Ubicamos las experiencias de trabajo asociativo autogestionado para la producción de bienes y servicios en zonas rurales y semirrurales del sur, suroriente y península de Yucatán de México, dentro del espacio heterogéneo y en construcción de la economía social y solidaria. En un primer boceto sobre la extensión, características, actores y sujetos involucrados, analizamos cifras absolutas y relativas, así como la génesis, dinámica y vinculación actual e histórica con otros actores y sujetos sociales. Nuestra búsqueda es contribuir al debate sobre el desarrollo de la economía social y solidaria en México y América Latina, al cuestionar la potencialidad de estas prácticas para gestar otras economías, políticas y subjetividades.

\section{Palabras clave autores:}

trabajo asociativo para la producción; autogestión; economía social y solidaria; construcción social de alternativas; México

\section{Palabras clave descriptores:}

cooperativas; cooperativas de productores; México

\footnotetext{
*Este artículo hace parte del proyecto Respuestas y experiencias de innovación social ante la crisis estructural del empleo asalariado, financiado por la convocatoria Ciencia Básica de Conacyt, México.

**Doctora en Ciencia Social con Especialidad en Sociología. Investigadora Titular, departamento de Sociedad, Cultura y Salud de El Colegio de la Frontera Sur. Correo electrónico: magracia@ecosur.mx ${ }^{* * *}$ Doctor en Ciencias Políticas y Sociales. Investigador Titular, departamento de Sociedad, Cultura y Salud de El Colegio de la Frontera Sur. Correo electrónico: jhorbath@ecosur.mx
} 


\title{
A Journey Through the Experiences of Self-Managed Partnership Working in Southern Mexico
}

\begin{abstract}
The experiences of self-managed partnership working for producing goods and services in rural and semi-rural areas of the south, southeastern and the Yucatan peninsula of Mexico, are understood within the heterogeneous and ongoing space of social and supportive economy. In a first sketch on the extent, the characteristics, the actors and the subjects involved, we analyze absolute and relative figures, as well as the genesis, the dynamics and the current and historical linkages with other actors and social subjects. Our quest is to contribute to the debate on the development of the social and supportive economy in Mexico and Latin America, and to question the potential of these practices for gestating other economies, policies and subjectivities.
\end{abstract}

Keywords author:

associative work for production; self-management; social and supportive economy; social construction of alternatives; Mexico

Keywords plus:

cooperative societies; producer cooperatives; Mexico

\section{Un parcours par les expériences de travail en partenariat autogéré au sud de Mexique}

\section{Résumé}

On situe les expériences de travail en partenariat autogéré pour la production de biens et de services dans des zones rurales et semi-rurales du sud, du sud-est et de la péninsule de Yucatan de Mexique, à l'intérieur du espace hétérogène et en construction de l'économie sociale et solidaire. Dans un premier esquisse sur l'extension, les caractéristiques, les participants, et les personnes impliquées, on analyse les chiffres absolus et relatifs, aussi la genèse, la dynamique et le lien actuel et historique avec d'autres participants et des personnes de la société. Notre recherche est celle de contribuer au débat sur le développement de l'économie sociale et solidaire au Mexique et en Amérique Latine, au moment de nous demander sur la potentialité de ces pratiques pour concevoir d'autres économies, politiques et subjectivités.

\section{Mots-clés auteur:}

travail en partenariat pour la production; autogestion; économie sociale et solidaire; construction sociale d'alternatives; Mexique

Mots-clés descripteur:

coopératives; coopératives de production; Mexique 


\section{Introducción}

Este artículo se interesa por las experiencias de trabajo asociativo autogestionado emergentes en México y su análisis crítico en el contexto de otras iniciativas de este orden que van surgiendo en América Latina. Ubicamos estas experiencias en los grupos de trabajadoras y trabajadores que autogestionan actividades de producción de bienes o servicios, cuya finalidad es la satisfacción de sus necesidades y no la acumulación de capital.

A partir de los avances de una investigación en curso financiada por el Consejo Nacional de Ciencia y Tecnología (Conacyt) de México nos interrogamos sobre la posibilidad de estos grupos para generar trabajo, ingresos y ciudadanía en contextos de crisis económicas recurrentes, pérdida de empleo estable, precarización de las condiciones de trabajo y deterioro de los derechos sociales y políticos conquistados décadas atrás por diversos colectivos sociales. ¿Qué tipo de alternativas representan? ¿Qué tipo de actores y sujetos colectivos emergen de éstas?

Más que dar respuestas acabadas a esta interrogante general, partimos de allí para orientar un recorrido exploratorio por las experiencias del sur de México. En el primer apartado presentamos la perspectiva teórico-metodológica para conceptualizar las experiencias estudiadas y generar las herramientas de producción de datos. En la sección de resultados, presentamos datos generales provenientes de fuentes secundarias para cuantificar el universo a escala nacional, y analizamos los datos producidos fundamentalmente a partir de fuentes primarias por subregiones. En el apartado de conclusiones, reflexionamos sobre los resultados y situamos las experiencias en el campo nacional de la economía social y solidaria.

\section{Perspectiva teórica y metodología}

\section{I.I. Las experiencias de trabajo asociativo en el campo de la economía social y solidaria}

Ubicamos las formas asociativas en el espacio de la economía social y solidaria, donde confluyen una multiplicidad de propuestas teórico-conceptuales y de experiencias concretas que se retroalimentan mutuamente de diversas maneras y con distintos grados de sinergias. Este espacio tiene desarrollos analíticos diferenciales en los distintos países y regiones de América Latina y se nutre e interactúa con experiencias y propuestas teóricas de otros lugares del mundo, en particular, las desarrolladas en el mundo francófono (Francia, Bélgica y Canadá). 
En nuestra región aparecen organizaciones de la sociedad civil de base y apoyo; nuevos movimientos sociales que incluyen prácticas productivas y de intercambio y comercialización; empresas recuperadas y autogestionadas por sus trabajadores y trabajadoras fundamentalmente en Argentina, Brasil, Uruguay y Venezuela; experiencias asociadas con la problemática del hábitat popular; redes de comercio justo, mercados (tianguis) orgánicos y solidarios y redes de consumo responsable; emprendimientos económicos populares mercantiles y no mercantiles; cooperativas de producción, de vivienda, de servicios públicos, de ahorro y préstamo y de consumo; finanzas solidarias, monedas sociales, redes de trueque y ayuda mutua y de comercialización/distribución de bienes y servicios; asociaciones vecinales; experiencias de presupuesto participativo, entre otros.

Se trata de un espacio heterogéneo pues las experiencias son diversas, como los actores que las protagonizan, sus lógicas, alcances y su capacidad de plantear alternativas a los modelos de desarrollo que se han venido aplicando con poco éxito en el país y en la región.

Como espacio en construcción, sucede con algo similar a lo que ocurre cuando se busca estudiar un movimiento social: no puede ser equiparado con un actor empírico pues, básicamente, es una construcción analítica que tiene fuertes componentes "utópicos” y ético-políticos, dado que persigue la construcción de posibles, de alternativas, de emancipaciones. En el caso de la economía social y solidaria el vínculo con el pensamiento utópico también se relaciona con las utopías católicas inspiradas en las comunidades cristianas primitivas, por lo cual "los conceptos morales aplicados a la economía por parte de la iglesia aparecen en todo el continente [recuperando, por ejemplo] al Comercio Justo como propuesta de la comercialización solidaria" (Collin, 20I2, pp. 233-234), y con nexos particularmente estrechos con las comunidades eclesiales de base y la Teología de la Liberación. Al estudiar, específicamente, el caso mexicano, Laura Collin (20I2) expresa la hipótesis de que la propuesta solidaria, menos urticante que la liberacionista, ha permitido la expresión de los restos de la Teología de la Liberación que permanecieron en estado larvario durante años y que vuelven a emerger a la luz pública "[sobre todo] por las condiciones generadas por el neoliberalismo".

Consideramos que el campo de la economía social y solidaria no es un sector o subsector de la economía que pueda ser mensurado. Es una imagen deseable, dinámica, en construcción, que además se entrecruza con propuestas emergentes como el buen vivir y cuya función es, justamente, la de ser una anticipación imaginativa, una imagen que se construye mediante una serie de procesos de 
experimentación social de prácticas productivas, políticas, económicas, simbólicas (Gracia, 20II), que buscan, implícita o explícitamente, ser alternativas a la economía y política capitalistas, aunque desde distintos conceptos y diferencias de grado.

Son también prácticas que pueden ser pensadas como políticas de la posibilidad económica (Gibson y Graham, 20II), porque tienen la función y nos invitan a considerar aquellas opciones que fueron marginadas o que ni siquiera se han intentado (Santos, 2000, en Santos, 20II, p. I7), lo que amplía el campo de lo posible y, al mismo tiempo, pone en duda la separación tajante entre realidad y utopía.

Existe una diversidad de planteamientos teórico-metodológicos y de proyectos vinculados. Principalmente se distinguen las siguientes propuestas: Economía Solidaria (Singer, 2002; Gaiger, 2004; Razeto, 1993); Social y Solidaria (Laville, 2004); Autogestiva ${ }^{4}$ (Albuquerque, 2004); Economía de Trabajo (Coraggio, 2004, 20II); Popular (Sarria Icaza y Tiriba, 2003); Digna y Sustentable (López Llera, 2005). No solo existen diferencias entre estas aproximaciones sino que, además, cada una es dinámica en sí misma. Más allá de las diferencias, los planteamientos de estos autores no solo tienen en común el diagnóstico sobre la insuficiencia del mercado capitalista para integrar a amplios sectores de la población, sino también la necesidad, a la que nos adherimos, de pensar "lo económico" desde un horizonte más amplio que el de la ciencia económica y su teoría hegemónica (neoclásica).

En este sentido, hay una pretensión teórica de gestar una "teoría económica comprensiva” (Razeto, 1993, 2002) que retome los aportes de la sociología, la antropología y la historia económica, bajo la convicción epistemológica de que "las economías reales no pueden encararse desde una disciplina pretendidamente universal (...) que además se rige por un paradigma epistemológico positivista que de todas maneras no respeta" (Coraggio, 2002, p. I). Al recuperar los aportes de la antropología económica sustantivista (Polanyi, 1957; 2003), autores como Laville (2009) plantean como síntesis la propuesta de "economía plural” que, frente al reduccionismo de la corriente formalista (hegemónica), busca rescatar la diversidad de principios y prácticas que rigen las instituciones económicas, pues remiten a las relaciones de interdependencia entre las personas y la naturaleza.

A pesar de su visión crítica dentro de las ciencias económicas, distinguimos en algunos de estos planteamientos la tendencia a caracterizar las experiencias con predominio de aspectos económicos como si se pudiera crear "otra" economía, sin crear al mismo tiempo "otra" política, "otro" Estado, otra sociedad (Gracia, 20II;

4 Autogestionada. 
Marañón y López, 2010). En este sentido, consideramos fundamental abarcar en el análisis las dimensiones económicas, políticas y culturales, al integrar el problema del poder y la dominación, y cuestionar de manera epistemológica la separación entre lo económico, lo político y lo social evidente, desde la formación de las ciencias sociales y su separación de las humanidades (Wallerstein, 2003; Santos, 2009).

\section{I.2. Cambios en el conflicto y en la acción colectiva: el (re) surgimiento de la sociedad civil y la emergencia de la economía social y solidaria en México}

Si la historia muestra que los movimientos fueron concebidos, sobre todo, desde una perspectiva de acción política en su confrontación con el Estado, los movimientos emergentes parecen no estar interesados en la toma del poder. Como parte de las trasformaciones en los rasgos de la acción colectiva, desde diversas perspectivas analíticas se utiliza la noción de sociedad civil, cuyo resurgimiento se vincula tanto con las transformaciones en el régimen de acumulación y del Estado-nación, como con la disminución del peso relativo de la clase obrera y la crisis de la democracia representativa.

En el caso de los países del cono Sur y de Europa del Este, la emergencia de la sociedad civil tuvo que ver con el surgimiento de organizaciones comprometidas con los procesos de democratización luego de las dictaduras militares y los regímenes de socialismo real; en México, las organizaciones de la sociedad civil -muchas de las cuales se habían constituido en los años sesenta - se visibilizaron después del terremoto de 1985 , con su labor de apoyo y canalización de créditos internacionales cuando se convirtieron en interlocutoras del Gobierno.

En términos teóricos, el concepto de sociedad civil es sumamente ambiguo pues se utiliza tanto para defender la autonomía y el poder-hacer de asociaciones y colectivos, como para justificar programas de ajuste estructural que les transfieren funciones supletorias que otrora desempeñaba el Estado.

Una de las perspectivas principales de la sociedad civil es la liberal, que la define como “tercer sector", diferente al Estado (primer sector) y al mercado (segundo sector). Dentro de esta perspectiva, la tradición anglosajona restringe la utilización del término a las "organizaciones sin fines de lucro", mientras que la tradición europea la amplía al incluir las cooperativas y mutuales. Esta propuesta concibe al mercado como la solución de los problemas sociales y piensa que el Estado o la sociedad civil tienen que intervenir ante sus “fallas”, mediante la creación de 
opciones y ofertas para los sectores pobres que quedan fuera del mercado, lo que constituye más bien medidas asistencialistas desde una visión atomizada.

Otras perspectivas, que cuestionan la definición de “tercer sector", retoman nociones de sociedad civil como la de Antonio Gramsci (1986), quien la definió como el terreno en que se disputan relaciones de fuerza entre polos sociales antagónicos, donde es posible construir la hegemonía o consenso y también la contra-hegemonía o el poder popular. Desde esta visión, la sociedad civil no se opone de forma dicotómica al Estado, sino que forma parte de la noción de Estado ampliado que incluye tanto la sociedad civil como la sociedad política.

Las experiencias de trabajo asociativo autogestionado que estudiamos son dinámicas y forman parte de un conjunto de actores heterogéneos que participan de la lucha por la definición legítima del campo de la economía social y solidaria, de su identidad y de las relaciones con otros actores regionales, nacionales e internacionales.

En México la economía social tiene una larga tradición histórica en el cooperativismo, el mutualismo, los movimientos campesinos e indígenas, así como en el sindicalismo democrático y el movimiento urbano popular (Rojas, 2013, p. 150); sin embargo, si estudiamos la economía social y solidaria en términos de movimiento podemos situar su emergencia con el resurgimiento de la sociedad civil y con las distintas respuestas encaminadas a enfrentar la crisis del trabajo asalariado, el corporativismo y el paternalismo propio de la cultura política imperante, mediante prácticas autogestionadas que reivindican la autonomía y se organizan a partir de formas de democracia directa.

A partir de esas prácticas y luchas surgió en 1983 la reforma al Artículo 25 de la Constitución Política de México, que reconoce la existencia del denominado Sector Social de la Economía dentro del cual engloba a "los ejidos, organizaciones de trabajadores, cooperativas, comunidades, empresas que pertenezcan mayoritaria y exclusivamente a los trabajadores y en general, todas las formas de organización social para la producción, distribución y consumo de bienes y servicios socialmente necesarios”. Aquí se asume que el Estado debe promover y fomentar este sector.

\section{I.3. Universo de estudio y herramientas metodológicas}

Más que centrarnos en las organizaciones agrarias o en el sector cooperativo, por ejemplo, decidimos delimitar nuestro universo de manera amplia a partir de las experiencias de trabajo asociativo autogestionado ubicadas en zonas rurales, semirrurales y urbanas, encaminadas a producir bienes (del sector primario o secundario) y servicios 
para intercambiarlos en el mercado capitalista, en mercados o redes de comercio justo, o para complementar el consumo final del grupo o comunidad cuya lógica está íntimamente relacionada con las unidades domésticas de sus protagonistas. Con ello buscamos captar la heterogeneidad y las distintas lógicas subyacentes.

Se trata de organizaciones que privilegian lógicas reproductivas y no la acumulación del capital y en las que predominan (no sin tensión y diversas contradicciones materiales y subjetivas) las relaciones sociales de cooperación libre, horizontal y autónoma (esté o no cristalizada jurídicamente). Estas relaciones sociales se constituyen para la producción basada en principios de reciprocidad-solidaridad e igualdad, y en el ejercicio democrático de los poderes de decisión politica (se incluye el control y la rendición de cuentas) sobre la organización del trabajo, la apropiación y distribución de los productos de ese trabajo, así como sobre las vinculaciones con el Estado y otros actores sociales y políticos.

Para comenzar esta tarea, emprendimos un estudio de carácter exploratorio que combina herramientas metodológicas de la tradición cualitativa y cuantitativa, aunque con cierto predominio de las primeras. Con el fin de abarcar de forma tentativa este universo y seleccionar, en una segunda instancia del proyecto, casos para estudiar en profundidad, efectuamos un directorio provisional.

Esta selección, realizada entre enero y agosto de 20I2, partió de los siguientes criterios: a) información primaria proveniente de consultas, entrevistas semiestructuradas (Io) y en profundidad (7), realizadas a participantes de los grupos de trabajo asociado, a referentes clave de organizaciones de la sociedad civil organizada y a funcionarios públicos; b) información primaria surgida de recorridos de campo, visita y observación no sistemática de las principales experiencias de algunas zonas de las subregiones de estudio; y c) información secundaria proveniente de la revisión de la literatura y del análisis de la información del Directorio Estadístico Nacional de Unidades Económicas (Deneu) del Inegi, información que cruzamos con nuestros datos primarios. A continuación presentamos un boceto de nuestro universo de análisis que se encuentra invisibilizado en las estadísticas oficiales.

\section{Resultados: las experiencias de trabajo asociativo en las regiones de estudio}

Mostrar cuantitativamente el denominado sector social de la economía en México, en el marco del resto de sectores de la economía que aparecen en las estadísticas oficiales, es un gran reto. A pesar de que, como observáramos antes, la propia 
Constitución reconoce y consagra la existencia de un sistema de economía mixta (integrado por las organizaciones y empresas de la economía pública, la economía privada y la economía social), en realidad no existe una política de Estado abocada a la materia, lo cual se refleja en los instrumentos de clasificación y medición estatal. Al tomar como base los censos económicos y las encuestas, no es posible visibilizar a los sectores sociales de la economía de manera adecuada, ya que no tienen un diseño que permita captarla y delimitarla.

Lo anterior significa que desde los datos oficiales solo se puede hacer una aproximación a la magnitud de los distintos subgrupos. Las principales figuras jurídicas que adoptan estas organizaciones son las cooperativas y sociedades de solidaridad social (SSS); las organizaciones de tipo agrario en las que existe un régimen colectivo de tenencia (ejidos, comunidades agrarias y sus organismos de integración de segundo y tercer nivel: uniones de ejidos y comunidades, y asociaciones rurales de interés colectivo); y las organizaciones de la sociedad civil (OSC) que apoyan a las distintas organizaciones de base.

A efectos de proporcionar cifras nacionales de los principales subgrupos, señalamos que en el año 2009, de las 273072 unidades de sociedades mercantiles, 6416 eran cooperativas, es decir, 2.35\% del universo de sociedades mercantiles (Instituto Nacional de Geografía y Estadística [Inegi], 20го). Estos datos son intermedios respecto a otra fuente oficial (el Deneu del Inegi') que incluía para el año 2012 a 2586 sociedades y una aproximación de la Cámara de Diputados de la Nación de 2005, que las calculaba en alrededor de 13316 (Rojas, 2006, p. 134).

Las organizaciones de tipo agrario son las que tienen más peso en el sector social, en tanto presentan aproximadamente 30000 entidades asociativas (Rojas, 20I3, p. 152). A partir de los datos disponibles de los censos ejidales de $200 \mathrm{I}$ y 2007, una cuestión que sobresale es el aumento del número de ejidos en $\mathbf{2} .7 \%$ superior al número de ejidatarios que se incrementó en $8.7 \%$. Esto se debe más a la fragmentación de ejidos que a la ejidos formados en nuevas tierras, pues la política agraria ha ido abandonando el fomento y protección de esta forma social (Inegi, 2006; 2010).

De acuerdo con el Registro Federal llevado por el Instituto Nacional de Desarrollo Social (Indesol), en 2010 las OSC eran 12 602, en contraste con las 9013 que arrojó el censo de 2000 realizado por UAMI-Indesol, lo que muestra un incremento de $39.2 \%$ en esta década. Un problema que tiene la información, es que la Ley de Fomento a las Actividades Realizadas por OSC reúne a todas las

5 Recuperado el 7 de julio de 20r2, de http://www.inegi.org.mx/Sistemas/denue/Default.aspx 
organizaciones y no las distingue según las actividades que realizan y la población a la que se dirigen o con la que trabajan.

\section{I Península de Yucatán}

La península de Yucatán, situada en el sureste de México, está formada por tres estados, Yucatán, Campeche y Quintana Roo. El estado más joven es Quintana Roo, que hace apenas cinco décadas era territorio nacional ${ }^{6}$ y tiene actualmente más de un millón de habitantes, I2\% de los cuales es de origen rural. Yucatán, estado que nombra a la península, tiene cerca de dos millones de habitantes, cuenta con $16 \%$ de población rural, y Campeche cuenta con casi 900000 habitantes, $25 \%$ de origen rural.

A partir del trabajo realizado para la confección del directorio pudimos identificar y constatar la existencia de alrededor de 200 proyectos de trabajo asociativo, organizados en su mayoría en cooperativas (algunas de ellas funcionan tanto en propiedades comunales como ejidales) y situados en los municipios con mayor población indígena. La mayoría de estos proyectos ofrecen productos y servicios en el mercado capitalista, muchos de ellos mediante organizaciones de Comercio Justo que facilitan la exportación. Esto los deja en fuerte dependencia de la demanda, las fluctuaciones del mercado y los precios internacionales. Sus protagonistas, inmersos en una economía campesina que ha ido trasmutando, por su mayor vulnerabilidad ante los cambios climáticos y las políticas de liberalización del comercio, combinan los ingresos que reciben por la actividad asociativa con lo que se produce en la unidad doméstica -agricultura en la "milpa" para el autoconsumo-, el cuidado de animales de traspatio, la silvicultura, pesca en algunos casos y ganadería en otros.

Hemos detectado solo unos pocos e incipientes tianguis (mercados) para intercambio de productos que plantean lógicas agroecológicas, orgánicas y solidarias. En esta zona existe una creciente migración de jóvenes (sobre todo hombres) a las zonas de gran demanda turística y hacia Estados Unidos. En términos generales, se trata de una región con creciente vocación turística pues en ella se ubican Cancún -uno de los polos de mayor desarrollo en este sector en el mundo- y la Riviera Maya costera, que abarca desde Puerto Morelos hasta Tulum y la isla de Cozumel.

6 A diferencia de los "estados" de la Federación, los "territorios federales" estaban menos poblados y en ellos no había elecciones de gobernador sino que este era nombrado por el Presidente de la República.

7 La milpa es un agroecosistema mesoamericano. Se denomina así tanto al espacio físico (la tierra o parcela) como lo que allí se siembra, principalmente, maíz, frijol y calabaza, complementados por el chile en algunas regiones. 
Estos puntos ofrecen zonas exóticas y exclusivas en un espacio de gran complejidad y fragilidad ecológica, cuyo gran impacto ambiental contrasta con la escasa derrama económica hacia las economías locales y regionales; por tanto, no se evidencia en el bienestar de las poblaciones que migran desde las zonas indígenas rurales -inclusive desde los municipios autónomos zapatistas de Chiapas- para obtener bajos niveles de remuneración y condiciones de trabajo, muchas veces extenuantes, en un espacio que, además, ha sido identificado reiteradamente como altamente discriminatorio y estigmatizante para las poblaciones originarias de la zona.

Toda la península destaca por su diversidad y riqueza ecológica, evidente en sus seis reservas de biósfera (dos en cada uno de los estados), siete parques naturales y tres áreas protegidas de flora y fauna. De allí que $25 \%$ de las cooperativas y grupos que detectamos en esta región están organizados para realizar actividades vinculadas con el turismo ecológico o ecoturismo, ya sea dentro de las reservas o áreas protegidas donde habitan pescadores y comunidades indígenas o zonas aledañas. Estas actividades se desarrollan sobre todo en la zona norte del estado de Quintana Roo (municipio Lázaro Cárdenas aledaño al municipio Benito Juárez, donde se encuentra Cancún), algunas en la zona norte de Yucatán y en el suroeste de Campeche.

Este trabajo no solo involucra una serie de elementos organizativos, económicos y comerciales sino que aborda aspectos de manejo y conservación de recursos naturales. En su mayoría, se trata de experiencias de formación reciente (surgieron luego del año 2000), por lo que aún no están del todo consolidadas o articuladas en redes o conectadas con organizaciones regionales o nacionales de economía solidaria. No obstante, estas sí reciben apoyo de distintas organizaciones de la sociedad civil que trabajan en la zona, y de programas de organismos internacionales de cooperación como el Compact del PNUD.

Una de las excepciones en términos de articulación, lo constituye la denominada Puerta Verde, promovida por seis comunidades rurales e indígenas mayas, que involucra dos Áreas Naturales Protegidas de carácter federal, Yum Balam (en Quintana Roo) y Otoch Maax Yetel Koh (en Yucatán). En mucho menor medida, también encontramos experiencias de turismo cultural sobre todo en Yucatán y Campeche, y más recientemente varios grupos de pobladores mayas se están organizando de manera autogestionada para desarrollar proyectos de este tipo en el municipio de Carrillo Puerto, en el centro del estado de Quintana Roo.

Muchas de estas experiencias han surgido o cuentan con el apoyo del Programa Turismo Alternativo en Zonas Indígenas que ejecuta la Comisión Nacional para el Desarrollo de los Pueblos Indígenas (CDI). Si bien -según distintos casos- 
en algunos aspectos el programa es relevante para ampliar la oferta de servicios, y en ocasiones incluir alojamiento (como en las experiencias del municipio de Nuevo Durango al norte del Estado de Quintana Roo), no alcanza a ser una oferta autosuficiente en un mercado sumamente competitivo donde, además, existen fuertes presiones sobre la tierra.

Debido a lo anterior, con frecuencia los proyectos terminan explotados por grandes empresarios que alquilan zonas comunitarias y ejidos para desarrollar una serie de servicios vinculados con el ecoturismo y turismo de aventura. En términos de apropiación y búsqueda de autonomía, detectamos una experiencia (Punta Laguna) protagonizada por jóvenes mayas en el norte del estado de Yucatán, que aprendieron a hablar inglés directamente de "la maya” sin pasar por el español.

La actividad pesquera es sumamente importante en toda la península; en esta destacan las cooperativas que se han venido formando, sobre todo, a partir de los años cincuenta del siglo pasado y que tienen concesión de pesca o solo permiso en ciertos casos (algunas pescan en áreas protegidas). En nuestro directorio, $35 \%$ de los grupos se concentra en el sector pesquero. Si bien la pesca de langosta a escala nacional representa solo $2 \%$ de la pesca total, en términos de valor comercial es uno de los productos más importantes. En la península de Yucatán la producción actual de langosta es de 7II toneladas en promedio, producción en la cual los estados de Yucatán y Quintana Roo han registrado volúmenes similares. En los registros de la Comisión Nacional de Acuacultura y Pesca (Conapesca, 2010), la langosta aportaba $33 \%$ del valor de producción pesquera total del estado de Quintana Roo.

Al ser una zona con la mayor cantidad de selva y bosques del país, también tienen gran importancia las actividades derivadas de su aprovechamiento como la apicultura, la producción maderera y la histórica explotación del chicle. En esta dirección hemos encontrado experiencias más consolidadas de productores de miel orgánica en el sur de Quintana Roo, cerca de Bacalar (Cooperativa Kabi Habin) que surgieron hace casi ya 20 años, y han enfrentado y superado momentos sumamente difíciles derivados de los destrozos de los huracanes que azotan a la región. Como otros productores de la zona, el grueso de la producción se efectúa a partir del Comercio Justo y se exporta a Europa. La mayoría de los socios apicultores también se dedican a la agricultura (algunos de ellos para el autoconsumo) y otros tienen ganado.

Por lo general, la organización del trabajo es por grupo doméstico y durante la cosecha los productores asociados a la cooperativa recurren a conocidos o familiares (no socios). Un tema preocupante para los apicultores del sureste mexicano son 
transgénicos, ya que la introducción de esta siembra (detenida desde el año 2012 en la península pero no en el estado de Chiapas) representa una gran amenaza, debido a sus efectos en la salud pública y el impacto económico que tendría si se perdiera la calidad orgánica de la miel. De allí que este tema sea de importancia central y los grupos, redes y organizaciones han promovido diversas reuniones entre sí, con funcionarios públicos y con el sector académico.

En torno a la producción del chicle, a principios de los años noventa surgió una experiencia que hoy se denomina Consorcio Chiclero, e involucra 2000 productores que pertenecen a 46 cooperativas en los estados de Campeche y Quintana Roo. La iniciativa partió de la cooperativa Noh Bec con el propósito de rescatar la histórica actividad chiclera, por entonces en una crisis profunda. Esta experiencia se fomentó con recursos organizacionales del modelo de desarrollo que otrora había impulsado al sector (cooperativas y apoyo estatal), con un fuerte predominio de técnicos o profesionales vinculados al sector estatal, quienes se movían paralelamente como consultores y como promotores del sector social.

Si bien el consorcio sostiene en el discurso y en la práctica una mayor igualación en la distribución de ingresos y en la derrama económica a las comunidades, lo hace desde una lógica técnico-empresarial que piensa el cambio y las transformaciones más desde "arriba hacia abajo" que a partir de un trabajo desde la base que privilegie las relaciones y dimensiones pedagógico-culturales.

La participación de las mujeres en actividades asociativas comienza a cobrar mayor importancia pues, de forma gradual, se han ido involucrando en casos como la apicultura y el rubro artesanal e incluso han creado grupos exclusivos (mujeres indígenas en su mayoría). Ejemplo de ello es la cooperativa Melitzaak (la melipona que cura -maya-) en Felipe Carrillo Puerto, formada por un grupo de esposas de apicultores, que empezaron hace unos cuatro años a fabricar productos derivados de la miel y en el presente tienen más de roo productos, entre jabones, dulces y otros.

\subsection{Sur y Sureste}

De acuerdo con la regionalización utilizada, el Sur abarca los estados de Chiapas (con 4796580 habitantes), Oaxaca (con 3 80r 962 habitantes) y Guerrero (con 3388 768 habitantes); y la región Sureste, los estados de Veracruz (con 7643194 habitantes) y Tabasco (con 2238603 habitantes), todos con importantes concentraciones de población indígena, gran diversidad y riqueza en sus patrimonios culturales y ambientales, y también con importantes organizaciones y tradición de lucha. 
Pudimos identificar y constatar la existencia de aproximadamente 600 proyectos en el sur y 329 en el sureste, organizados en su mayoría en cooperativas, aunque también dentro de los ejidos, comunidades indígenas y luego nucleados en Unidos de Ejidos o Uniones de Comunidades. En la zona del istmo (Oaxaca), en la sierra norte y sur de Oaxaca y en los Altos de Chiapas, detectamos experiencias de cuidado de ganado en las comunidades autónomas zapatistas, de producción orgánica de café y de otros productos primarios (observamos la diversificación de esas actividades a partir del procesamiento de los productos primarios), la creación de cajas de crédito y ahorro, y el uso y conservación comunitaria de los recursos forestales.

En estas regiones, donde predomina la economía primaria y campesina, la organización en torno al proceso productivo del café ha sido de gran relevancia en la movilización y lucha de los movimientos indígenas. Podemos decir que las experiencias son diversas en cuanto a los pueblos y actores involucrados, pero más allá de sus especificidades locales, constituyen expresiones de las luchas por la independencia y la autonomía, esta última no solo en la autogestión productiva sino en la cultura y el autogobierno (Flores, Paré y Sarmiento, 1988).

Un eje de reclamo y lucha es y sigue siendo el control de las condiciones de producción y comercialización, por lo cual las acciones combinan demandas dirigidas al Estado (para solicitar créditos, asistencia técnica, regulación de precios o desarrollo de infraestructura) con la propia organización para paliar la falta de financiamiento productivo, así como los problemas derivados de la usura y de los intermediarios ("coyotes"). Debido a esto, $25 \%$ de los casos en el sur y $15 \%$ del sureste están representados en nuestro directorio por las cajas de ahorro popular.

Los elementos comunes a estas experiencias y que sobresalen en distintos casos es la composición indígena de los grupos y el apoyo que han tenido de la Pastoral Social de la iglesia, vinculado con el trabajo que la iglesia católica hizo desde los años sesenta con los indígenas. Esto se efectuó a partir de organizaciones como el Centro Nacional de Pastoral indígena (Cenapi, que ya no existe) y el Centro Nacional de Apoyo a las Misiones Indígenas (Cenami) que estimulan el desarrollo desde una visión integral, incluyen la dimensión subjetiva y cultural, y recuperan la medicina tradicional y la teología india. Asimismo, promueven la búsqueda de mejores condiciones económicas para la venta de sus productos mediante la producción orgánica y el Comercio Justo.

Entre los ejemplos más destacados encontramos la Unión de Ejidos Nahua Otomí y Tepehua (Veracruz); la Coordinadora Estatal de Productores de Café de Oaxaca (Cepco); Comunidades Campesinas en Camino (CCC), ubicada en la zona 
del istmo en Oaxaca, es una Sociedad de Solidaridad Social (SSS) con I000 socios productores, que sobresale por su diversificación económica (producción orgánica, procesamiento del aceite de ajonjolí y una caja de crédito y ahorro que tiene 4500 socios), y la notable importancia dedicada a la educación y capacitación; la Unión de Ejidos de la Selva (Chiapas); la Unión de Ejidos y Comunidades de Caficultores del Beneficio Majomut (Chiapas), que fue el antecedente de las actuales cooperativas cafetaleras en Chiapas; y la Coordinadora de Pequeños Productores de Café de Chiapas (Coopcafe), que agrupa a 36 organizaciones y 17000 campesinos que dedican una parte de su trabajo a la producción y comercialización de café orgánico.

Dentro del universo de organizaciones que pujan por la autonomía, encontramos una cooperativa surgida del grupo Las Abejas, fundado en diciembre de 1992, dos años antes del alzamiento zapatista, cuando los habitantes de la parroquia de San Pedro Chenalhó buscaron diferenciarse de los conflictos políticos, derivados de la gran polarización entre cardenistas y priístas, y abocarse a la defensa de los derechos humanos, en búsqueda de una justicia con dignidad en contra de la opresión y discriminación de los gobiernos.

Cuando ocurrió el alzamiento zapatista, el grupo Las Abejas creó una zona neutral del conflicto, aunque apoyó las demandas del movimiento en torno a la democratización, la seguridad en la tenencia de la tierra y los derechos indígenas de todas las etnias del país -posteriormente devino la demanda de autonomía-, pero sin coincidir en la toma de las armas como método para conseguirlas (Zamora, 2003). En el contexto del conflicto regional, una parte del grupo Las Abejas decidió formar la Unión de Productores Maya Vinic, que se registró legalmente el 29 de julio de 1999 como cooperativa cafetalera en los municipios de Chenalhó, Pantelhó y Charchihuitán. Esta unión tuvo como finalidad paliar la problemática de los pequeños cafeticultores del grupo, aquellos con propiedades menores a las dos hectáreas de cultivo. En la actualidad la cooperativa tiene 473 socios, productores de café, entre quienes se cuentan 40 mujeres.

En el mundo zapatista, la amplitud de las experiencias regionales de militantes del cooperativismo y la autonomía indígena, encuentra una ilustración sugerente en la consolidación de los "colectivos", es decir, las cooperativas de producción como alternativa económica en los municipios autónomos zapatistas de la selva Lacandona en Chiapas. Desde hace I8 años se ubican no solo en la dimensión de la resistencia abierta a los modos productivos capitalistas, sino que representan modalidades de carácter emancipador, al impulsar alternativas para procesos de desarrollo local y microrregional que solo se pueden superar al retomar iniciativas de transformación política y social a escala nacional (Cadena, 2005). 
En casi mil comunidades tzeltales, tsotsiles, choles y tojolabales, que pertenecen a la zona de la diócesis de San Cristóbal de Las Casas, la Ley Agraria Revolucionaria (1993) implica que las tierras "recuperadas" por el Ejército Zapatista de Liberación Nacional sean repartidas como propiedades colectivas, entre campesinos sin tierra y jornaleros agrícolas, para la formación de cooperativas campesinas y "colectivos" de producción agrícola y ganadera. En estas regiones rurales multiétnicas, donde la teología de la liberación india y el cooperativismo tuvieron impactos profundos en los años setenta y ochenta, se siguen creando hoy "colectivos" en los ejidos, rancherías y nuevos centros de población zapatista.

En la zona del estado de Tabasco hemos identificado organizaciones que se manifestaron más destacadas por su articulación entre lo económico, social y ambiental, con su preocupación y capacidad de respuesta y aprendizaje ante las crisis derivadas de fenómenos "naturales" (aunque los así llamados son en realidad el resultado de la interrelación compleja entre el ambiente y las formas de organización espacial de las sociedades). Entre estas se destacan las dirigidas a generar proyectos productivos en el contexto de las fuertes inundaciones que sufre el estado de Tabasco, que han llegado a afectar casi $80 \%$ de su territorio. Una de estas es la Asociación Civi I’xiktar Yinikos Aj' Juate Buka dedicada a promover la medicina tradicional, la acuicultura, la desalinización de los suelos y la producción de alimentos orgánicos. Otro caso muy significativo es la Sociedad Cooperativa Semillas de Solidaridad, constituida en febrero de 2009 a partir de la ejecución de un proyecto impulsado por la diócesis de Tabasco, con el propósito de reconstruir la agricultura campesina afectada por las inundaciones. La cooperativa se ha encargado de coordinar los esfuerzos de capacitación organizacional implementados en el estado de Tabasco y en abril de 20ro, la base social del proyecto incluía un total de 88 emprendimientos asociativos. En estos participaban alrededor de mil campesinos, con presencia en 15 de los 17 municipios del estado.

\section{Conclusiones: las experiencias de trabajo asociativo} en el sur de México a la luz de la formación del campo de la economía social y solidaria a escala nacional

Si bien cada una de las experiencias y organizaciones a las que hicimos referencia tienen sus peculiaridades y lógicas, y están circunscritas a contextos regionales con dinámicas complejas y específicas, también es fundamental observar que ellas 
no pueden ser cabalmente comprendidas si no son situadas en la cartografía de organizaciones y movimientos que ciertamente son nacionales, y que han aportado a la formación dinámica y en disputa del actual espacio de la economía social y solidaria.

A partir de los datos preliminares generados mediante las herramientas metodológicas utilizadas, se pueden clasificar las experiencias de trabajo asociativo exploradas dentro de tres grandes grupos de actores que operan en el espacio nacional: a) Grupos que predominantemente operan a partir de lógicas corporativo-estatales (solidaridad clientelista); b) Grupos que predominantemente se rigen a partir de lógicas filantrópicas (solidaridad filantrópica); c) Grupos que predominantemente funcionan a partir de lógicas autónomas (solidaridad democrática).

En los años posteriores a su creación en 2003, el Espacio Economía Solidaria (Ecosol) reúne actores clave en el impulso y seguimiento a los proyectos del campo de la economía solidaria. Señalamos también el protagonismo de la Coordinación Nacional de Uniones y Federaciones de Cooperativas que incluye a cooperativas de todo tipo, en especial, en el Distrito Federal. Durante la primera década del siglo XXI distintos representantes y organizaciones de las redes cooperativistas más activas se comprometieron abiertamente con grupos políticos de izquierda, como el Partido de la Revolución Democrática, la Otra Campaña Zapatista y el Foro Social Mundial.

En las regiones del sur y el centro del país -en particular, las que son indígenas y rurales- los militantes vinculados con los equipos pastorales, los movimientos agrarios y cooperativistas, recurren a modos de producir e intercambiar mediante una forma autonómica de organización. En estos se destacan los vínculos sociales generados por proyectos, en los cuales se reproducen valores y conocimientos basados en la comunalidad, la etnicidad y la reciprocidad.

La generación de excedentes proviene de actores comprometidos en una organización productiva, la cual está marcada por un sentimiento de pertenencia comunal, que reivindica la autonomía por medio de la toma de decisiones de forma colectiva, las faenas y la rotación de los cargos. Las experiencias cooperativistas en los pueblos indígenas parecen ser tributarias de una historicidad de relaciones complejas, mediante las cuales los sujetos en las luchas autonómicas han reproducido y se han apropiado de conocimientos y valores, (como la ética de la solidaridad y del trabajo colectivo), así como de la participación activa en su papel de ciudadanos y activistas culturales.

Las restricciones en el acceso al crédito, pero también a la educación agrícola y a la asesoría técnica son obstáculos que afectan los rendimientos y la consolidación de las cooperativas rurales y grupos de pequeños productores independientes. Ante 
este reto, una iniciativa metodológica de investigadores y alumnos de la Universidad Autónoma de Chapingo consiste en apoyar a redes de escuelas campesinas, donde se intercambian conocimientos sobre producción, gestión y política rural.

Como soporte de aprendizaje autónomo en nueve encuentros nacionales organizados desde el año 2003, el método de aprendizaje "de campesino a campesino" permite que interactúen representantes de las escuelas campesinas relacionadas con organizaciones indígenas de los estados del centro y el sur del país. Además de los aportes técnico-productivos comunes en este tipo de encuentro independiente, de formación de jóvenes y adultos indígenas, los intercambios son favorables a la difusión de prácticas sugerentes en materia de toma horizontal de las decisiones, de rotación de los socios en los puestos de trabajo y de no separación entre trabajo manual y trabajo intelectual.

Ninguno de estos tres agrupamientos es homogéneo y su carácter es tentativo. Están realizados según las lógicas predominantes, de allí que muchas veces las experiencias y agrupaciones estudiadas puedan tener rasgos de más de una, aunque se inclinan casi siempre por un tipo de solidaridad (clientelar, filantrópica o democratizante) que, ciertamente, va gestando proyectos socioproductivos, sujetos y actores sociales diferenciados.

\section{Referencias}

Albuquerque, P. (2004). Autogestión. En A. Cattani (Ed.), La otra economía (pp. 39-47). Buenos Aires: UNGS, Altamira, Fundación OSDE.

Cadena Barquín, F. (2005). De la economía popular a la economía de solidaridad. En B. Cadena (Coord.), De la economía popular a la economía de solidaridad. Itinerario de una búsqueda estratégica y metodológica para la construcción de otro mundo posible (pp. 13-49). México D. F.: El Colegio de Tlaxcala.

Collin, L. (2012). Economía solidaria: ¿capitalismo moralizado o movimiento contracultural? Tlaxcala: El Colegio de Tlaxcala, Conacyt.

Coraggio, J. L. (2002). Hacia un proyecto de economía social centrada en el trabajo: contribuciones de la antropología económica. Ponencia presentada en el Seminario Internacional Pekea, un saber político y ético para las actividades económicas. Santiago: Cepal.

Coraggio J. L. (2004). Economía del trabajo. En A. Cattani (Ed.), La otra economía (pp. 15I-163). Buenos Aires: UNGS, Altamira, Fundación OSDE. 
Coraggio J. L. (20II). La presencia de la economía social y solidaria (ESS) y su institucionalización en América Latina. Ponencia presentada en Estados Generales de la Economía Social y Solidaria. París.

Flores, G., Paré, L. y Sarmiento, S. (1988). Las voces del campo: movimiento campesino y política agraria, 1976-1984. México, D. F.: Siglo XXI Editores, Instituto de Investigaciones Sociales/UNAM.

Gaiger, L. I. (2004). Eficiencia sistémica. En A. Cattani (Ed.), La otra economía (pp. 213-220). Buenos Aires: UNGS, Altamira, Fundación OSDE.

Gibson, K. y Graham, J. (20II). Una política poscapitalista. Bogotá D. C.: Siglo del Hombre Editores.

Gracia, M. A. (20II). Fábricas de resistencia y recuperación social. Experiencias de antogestión del trabajo y la producción en Argentina. México D.F.: El Colegio de México.

Gramsci, A. (1986). Cuadernos de la cárcel (Tomos I a IV). México D. F.: Ediciones Era. Instituto Nacional de Geografía y Estadística [Inegi]. Anuarios Estatales de 2006 y 20ıo. México D. F.: Tesorería General de los Estados y Registro Público de la Propiedad y del Comercio.

Laville, J. L. (2004). Economía social y solidaria. Una visión europea. Buenos Aires: Universidad Nacional de General Sarmiento, Fundación OSDE.

Laville, J. L. (2009). Economía Plural. En A. Cattani, J. Coraggio y J. Laville (Orgs.) Diccionario de la otra economía (pp. 157-162). Buenos Aires, UNGS, Altamira, Clacso. López Llera, L. (2005). Reticulación entre empresas autogestivas. El fin del dinero y la restauración del crédito social. En F. Cadena Barquín (Ed.), De la economía popular a la economía solidaria. Itinerario de una búsqueda estratégica y metodológica para otro mundo posible (pp. I8I-191). Tlaxcala: El Colegio de Tlaxcala.

Marañón, B. y López, D. (2010). Economía solidaria y sociedad alternativa en América Latina. Hacia una agenda de investigación desde la descolonialidad. Alternativ@s, 5(67). Recuperado el 3r de marzo de 2014, de http://www.tau.org.ar/ upload/89foc2b656cao2ff 45 ef6ra4f2e5bf24/ALTS66.pdf

Polanyi, K, (1957). The Economy as an Instituted Process. En K. Polanyi, C. M. Arensberg y H. W. Pearson (Eds.) Trade and Market in Ancient Empires (pp. 234-269). Nueva York: The Free Press.

Polanyi, K. (2003). La gran transformación. Los orígenes politicos y económicos de nuestro tiempo. México D. F.: Fondo de Cultura Económica.

Razeto, L. (1993). Los caminos de la economía de solidaridad. Santiago: Vivarium. Razeto, L. (2002). La economía de la solidaridad: Hacia una nueva civilización latinoamericana. Ponencia presentada en el II Foro Social Mundial de Porto 
Alegre, en el Seminario sobre la Economía de Solidaridad, Porto Alegre, Brasil. Recuperado el or de febrero de 2002, de http://www.luisrazeto.net/content/ econom\%C3\%ADa-de-solidaridad-y-profundizaci\%C $\% \% B 3 n$-de-la-democraciapara-una-nueva-civilizaci $\% \mathrm{C}_{3} \% \mathrm{~B} 3 \mathrm{n}$

Rojas, J. J. (2006). Análisis del panorama asociativo presente en el llamado sector social de la economía mexicana. En J. Pérez de Uralde y M. Radrigán Rubio, La economía social en Iberoamérica. Un acercamiento a su realidad. Volumen II (pp. II3-I54). Madrid: Fundación Iberoamericana de Economía Social.

Rojas, J. J. (2013). Fortalezas y debilidades de la economía social en México y sus perspectivas de cara a los retos que impone el modelo neoliberal. En L. Oulhaj (Coord.), Miradas sobre la economía social y solidaria en México (pp. I49-159). Puebla: Universidad Iberoamericana de Puebla.

Santos, B. d. S. y Gandarilla Salgado, J. G. (2009). Una epistemología del sur la reinvención del conocimiento y la emancipación social. México, D. F.: Siglo XXI Editores, Clacso.

Santos, B. (20II). Producir para vivir. Los caminos de la producción no capitalista. México D. F.: Fondo de Cultura Económica.

Sarria Icaza, A. y Tiriba, L. (2003). Economía Popular. A outra economia. San Pablo: Editora Veraz.

Singer, P. (2002). A recente ressurreicao da economia solidária no Brasil. En B. Santos (Org.) Produzir para viver. Os caminhos da producao nao capitalista, (pp. 8I-I29). Rio de Janeiro: Civilizacao Brasileira.

Wallerstein, I. (2003). Abrir las ciencias sociales. México D. F.: Siglo XXI Editores, Centro de Investigaciones Interdisciplinarias en Ciencias y Humanidades/ UNAM.

Zamora, C. (2003). Xnix Cajbe (flor de café). El proceso organizativo de Las Abejas y Maya Vinic y su impacto en el espacio social regional en los Altos de Chiapas. (Tesis no publicada de Maestría en Estudios Regionales), Instituto Mora. Recuperado el 31 de marzo de 20I4, de http://www.academia.edu/5373340/ Tesis_de_maestria_en_estudios_regionales_XNIX_CAJBE_FLOR_DE_CAFE__EL PROCESO_ORGANIZATIVO_DE_LAS_ABEJAS_Y_MAYA_VINIC_Y_SU_IMPACTO_EN_EL_ ESPACIO_SOCIAL_REGIONAL_EN_LOS_ALTOS_DE_CHIAPAS 